\title{
Evaluación y selección participativa de híbridos de sandía [Citrullus lanatus (Thunb.) Matsum y
}

Nakai] en invernadero

\section{Evaluation and participatory selection of watermelon hybrids [Citrullus lanatus (Thunb.) Matsum}

\section{and Nakai] in greenhouse}

\author{
Gabriel-Ortega Julio $^{1 *(D)}$, Barahona-Cajape Nora ${ }^{2}(\mathbb{D})$ Burgos-López Gema $^{3}(\mathbb{D})$, Ayón-Villao Fernando $^{1(D)}$, \\ Narváez-Campana Washington ${ }^{1}(\mathbb{D})$, Vera Tumbaco Máximo ${ }^{1}$
}

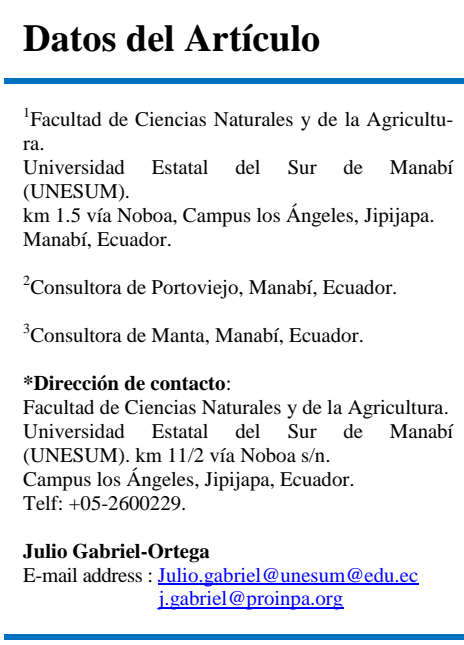

Palabras clave:

Criterios,

preferencia,

selección,

evaluación participativa,

consumidores,

cadena de va

J. Selva Andina Res. Soc.

2021; 12(1):52-63.

ID del artículo: 144/JSARS/2020

\section{Historial del artículo.}

Recibido agosto 2020

Devuelto noviembre 2020

Aceptado diciembre 2020

Disponible en línea, febrero 2021.

\section{Editado por: \\ Selva Andina \\ Research Society}

\section{Keywords:}

Criteria,

preference,

selection,

participatory evaluation,

consumers,

value chain.

\section{Resumen}

La presente investigación se realizó en Puerto La Boca, provincia Manabí, Ecuador, en el año 2019, con el objetivo de evaluar y seleccionar participativamente híbridos de sandía en invernadero. Para el desarrollo de esta investigación se implementó dos experimentos: en el primer experimento se realizó una parcela en invernadero bajo un diseño completamente aleatorizado con tres tratamientos $\left(\mathrm{T}_{1}\right.$ : Quetzalí, $\mathrm{T}_{2}$ : Champagne y $\mathrm{T}_{3}$ : Vanessa) y en el segundo experimento, se evaluó y seleccionó los híbridos participativamente, considerando los criterios de preferencia de los actores de la cadena de valor de la sandía (consumidores). Este experimento fue analizado en un diseño completamente aleatorizado. Las variables de respuesta fueron: peso de frutos $(\mathrm{PF})(\mathrm{kg})$, volumen total de los frutos (VF) $\left(\mathrm{cm}^{3}\right)$, criterios de preferencia (color de fruto, color de carne, sabor, dulzor, olor y venta al mercado), datos pasaporte morfológico y una estimación económica. Los resultados expusieron que el híbrido Champagne fue el mejor, en características morfológicas, agronómicas y rendimiento, sin embargo, en los criterios de preferencia y venta, los consumidores eligieron al híbrido Vanessa como la mejor. El análisis de rentabilidad, mostró que los cultivares Champagne y Vanessa fueron rentables cuando fueron comercializados (B/C>1).

2021. Journal of the Selva Andina Research Society ${ }^{\circledR}$. Bolivia. Todos los derechos reservados.

\section{Abstract}

The present investigation was carried out in Puerto La Boca, Manabí province, Ecuador, in 2019, with the aim of participatory evaluation and selection of watermelon hybrids in the greenhouse. For the development of this research, two experiments were implemented: in the first experiment, a greenhouse plot was carried out under a completely randomized design with three treatments $\left(\mathrm{T}_{1}\right.$ : Quetzalí, $\mathrm{T}_{2}$ : Champagne and $\mathrm{T}_{3}$ : Vanessa) and in the second experiment, it was evaluated and selected the hybrids participatively, considering the preference criteria of the actors in the watermelon value chain (consumers). This experiment was analyzed in a completely randomized design. The response variables were: fruit weight $(\mathrm{FW})(\mathrm{kg})$, total fruit volume $(\mathrm{FV})\left(\mathrm{cm}^{3}\right)$, preference criteria (fruit color, meat color, flavor, sweetness, smell and sale to the market), morphological passport data and an economic estimate. The results showed that the Champagne hybrid was the best, in morphological, agronomic and performance characteristics; however, in the preference and sale criteria, consumers chose the Vanessa hybrid as the best. The profitability analysis showed that the Champagne and Vanessa cultivars were profitable when they were commercialized $(\mathrm{B} / \mathrm{C}>1)$. 


\section{Introducción}

La sandía (Citrullus lanatus), un cultivo diploide de 22 cromosomas, es una planta hortícola, herbácea y monoica cuyo origen se presume fue en África, donde aún hoy en día crecen en forma silvestre ${ }^{1-3}$. La sandía dulce presenta una secuencia de ADN polimórfico considerablemente menor que otros de su género ${ }^{4-11}$, lo que significa que se originó a partir de una población inicial.

Las sandías son cultivadas en las zonas más cálidas del mundo ${ }^{\underline{12}, 13}$, se siembran 3.4 millones de ha anualmente, con una producción superior a 102 millones de toneladas ${ }^{14}$.

En Ecuador la provincia con mayor superficie cultivada de sandía es Guayas con un $49 \%$, el segundo lugar está ocupado por Manabí con $44 \%$, luego los Galápagos y los Ríos que producen entre $1 \%$ y $3 \%$ respectivamente ${ }^{15}$.

El recinto Canta Gallo, ubicado a $4 \mathrm{~km}$ del filo costero del cantón Jipijapa, Provincia de Manabí, tiene un microclima particular para cultivar hortalizas y la mayoría de los productores cuentan con terrenos propios para esta actividad. La zona de Puerto la Boca aún se cultiva sandías en campo, pero en los últimos años hubo muchas pérdidas en las cosechas, debido a la alta incidencia y severidad causadas por los virus, trasmitidos por insectos vectores como la mosca blanca (Bemisia tabaci) y los áfidos (Myzus persicae $)^{\frac{16}{16}}$. Debido a lo mencionado, se volvió dificultoso el cultivo de sandía en campo, por lo que existe la necesidad de contar con nuevos cultivares de sandía que se adapten al manejo bajo invernadero. La zona cuenta con una asociación organizada con 60 familias de agricultores, que tienen 54 invernaderos de 500 a $1000 \mathrm{~m}^{2}$, que cubre un área de 2.74 ha, financiadas por el proyecto del Buen Vivir del Ministerio de Agricultura del Ecuador ${ }^{17}$.

53
La adaptación de la sandía a las diferentes características agroecológicas de forma general es la actitud suficiente de una planta para amoldarse a las condiciones del medio ambiente natural diferente al de su origen, finalmente es la concordancia entre las características morfológicas de un organismo con las características fisio-biológicas del medio, se dice que una planta se ha adaptado cuando su comportamiento agronómico y el rendimiento en su nuevo lugar son excelentes $\frac{18}{}$.

Por lo tanto, para obtener beneficios en la agricultura es necesario realizar cambios que permitan mejorar el nivel de vida del productor. El empleo de híbridos, se constituye en el principal insumo, como una alternativa tecnológica valida dentro del desarrollo y producción de esta actividad agrícola, no muy tradicional en el medio ${ }^{19}$.

Se debe destacar que existen diversos trabajos realizados sobre adaptación de híbridos de sandía en la provincia en Ecuador y particularmente en la provincia Manabí19-22 ${ }^{19}$, sin embargo, todos fueron realizados en campo, no bajo condiciones de invernadero. Esto marca una diferencia sustancial, debido a que si se logra la selección de un hibrido que se adapte las condiciones de este tipo de tecnologías podría ser potencial para producir la sandía durante todo el año.

Por lo que en el proceso de selección de cultivares mejorados, es fundamental la participación de actores de la cadena de valor de las hortalizas a través de una estrategia como es la selección participativa de nuevos cultivares (SPC), que consiste en lograr la participación de productores, comerciantes intermediarios, cadenas de supermercados, consumidores finales y otros ${ }^{23,24}$. La SPC se basa en la idea de que los agricultores y los otros actores, poseen co- 
nocimientos y habilidades importantes que podrían ser complementarios de los que poseen los fitomejoradores profesionales ${ }^{25}$. Los fitomejoradores son profesionales que adquirieron conocimientos y habilidades científicas que les dan la solvencia para seleccionar apropiadamente los genotipos a ser evaluados, pero esto se fortalece mucho más cuando en sus procesos de selección involucran a los agricultores $^{\frac{26}{6}}$ y otros actores de la cadena de valor de un producto $^{27}$.

La presente investigación tuvo como objetivo, evaluar, seleccionar convencional y participativamente el mejor híbrido de sandía para invernadero en la zona de Puerto La Boca, Ecuador.
La investigación se desarrolló en los meses de junio a octubre del 2019 en un invernadero de $1000 \mathrm{~m}^{2}$, con una humedad relativa mayor a $60 \%$ y temperatura promedio de $20^{\circ} \mathrm{C}$, ubicado en el Recinto Puerto la Boca de la Parroquia Puerto Cayo, Cantón Jipijapa $\left(1^{\circ} 18^{\prime} 20^{\prime \prime} \mathrm{S}, 80^{\circ} 45^{\prime} 42^{\prime \prime} \mathrm{O}\right.$ y altura de 53 $\mathrm{msnm}$ ), su clima posee una temperatura promedio anual de $24.8^{\circ} \mathrm{C}$, la precipitación promedio anual es de $298 \mathrm{~mm}$, concentrándose las lluvias en el mes de febrero, mientras que el mes más seco es agosto ${ }^{\underline{28}}$. La investigación fue desarrollada en la época de verano, el fotoperiodo es de al menos $12 \mathrm{~h} \mathrm{luz}^{\underline{28}}$.

Tratamientos y diseño experimental. Se utilizo tres híbridos de sandía [Citrullus lanatus (Thunb.) Matsum. y Nakai] (tabla 1).

\section{Materiales y métodos}

Tabla 1 Híbridos de sandía utilizados en la investigación ${ }^{29}$

\begin{tabular}{ccccc}
\hline Tratamiento & Cultivar & Ploidía & Empresa & Importadora \\
\hline $\mathbf{T}_{\mathbf{1}}$ & Quetzalí & Diploide $(2 \mathrm{n}=2 \mathrm{x}=22)$ & Syngenta & Importadora Alaska \\
$\mathbf{T}_{\mathbf{2}}$ & Champagne & Diploide $(2 \mathrm{n}=2 \mathrm{x}=22)$ & Syngenta & Importadora Alaska \\
$\mathbf{T}_{\mathbf{3}}$ & Vanessa & Triploide $(2 \mathrm{n}=3 \mathrm{x}=33)$ & Syngenta & Importadora Alaska \\
\hline
\end{tabular}

Los tratamientos concluyeron sin dificultad, no Datos pasaporte para morfología. Se evaluaron 17 observándose ningún problema fitosanitario diferente a los que comúnmente se presentan en la zona y las cuales fueron controladas con aplicaciones pertinentes.

La investigación fue realizada en dos experimentos: con los descriptores morfológicos del Instituto Nacional de Semilla $\frac{32}{\text {. }}$.

Experimento 1. Se implementó una parcela en invernadero en un diseño completamente aleatorio (DCA) con tres tratamientos ${ }^{30}$. Se eligieron en cada unidad experimental (UE) 30 plantas al azar por tratamientos.

Variables de respuesta. i) Peso de frutos (g). Se pesó los frutos cosechados de las plantas seleccionadas con una balanza. ii) Largo de frutos (cm). Se midió con la ayuda de una cinta métrica. iii) Ancho de frutos $(\mathrm{cm})$. Se evaluó en la parte media del fruto con una cinta métrica. iv) Volumen total de frutos $\left(\mathrm{cm}^{3}\right)$. Se utilizó la fórmula de Moreno ${ }^{31}$.

Tabla 2 Escala para la evaluación de preferencia del producto

\begin{tabular}{cc}
\hline Escala & Calificación \\
\hline 1 & Malo \\
2 & Regular \\
3 & Bueno \\
4 & Muy bueno \\
5 & Excelente \\
\hline
\end{tabular}

Experimento 2. Se evaluó los criterios de preferencia de los participantes en un diseño completamente aleatorio (DCA). La evaluación de preferencia fue realizada con una escala de 1 a $5^{\frac{33}{}}$ (tabla 2 ), las variables evaluadas fueron i) Color de piel (CPiel), ii) color de pulpa (CPulpa), iii) sabor (SFr), iv) dulzor 
(DFr), v) olor (OFr) y vi) venta al mercado (VMerc).

Análisis estadísticos. Previo al análisis de varianza (ANVA) para determinar los efectos fijos, se realizó un análisis de normalidad y homogeneidad de varianza. Se hicieron comparaciones de medias de los tratamientos mediante la prueba de Duncan al $\operatorname{Pr}<0.05$ de probabilidad ${ }^{30}$. El ANVA de los datos también sirvió para estimar los componentes de varianza para los efectos aleatorios y se analizó con el Proc GLM del SAS University ${ }^{34}$.

La preferencia por los híbridos fue analizada con el test de Kruskal-Wallis ${ }^{35}$ al $\mathrm{P}<0.01$ de probabilidad.

La correlación entre las variables evaluadas se realizó usando el coeficiente de Pearson ${ }^{30}$.

Manejo de la investigación. Los híbridos fueron en ocho platabandas de dos hileras por platabanda, con 55 plantas por hilera, cada hilera tuvo $33 \mathrm{~m}$ de largo y 110 plantas por platabanda, de los cuales se eligieron 30 plantas al azar por tratamiento. La distancia de siembra fue de $0.60 \mathrm{~m}$ entre plantas por hilera y 3 $\mathrm{m}$ entre platabandas. El número total de plantas fue de 880 plantas.

La preparación del suelo fue manual, se hizo la remoción y desterronado del suelo, y se formó las platabandas, donde se aplicó $75 \mathrm{~kg}$ de biocompost por hilera.

Para la germinación de la semilla se preparó sustrato con biocompost, hoja de guaba descompuesta y tierra del lugar, en una proporción 2:1:1. También se adicionó $10 \mathrm{~kg}$ de humus y $10 \mathrm{~g}$ de micorriza para prevenir el mal de almácigo causado principalmente por Fusarium sp. Una vez humedecido el sustrato a capacidad de campo, se llenó los alveolos de las bandejas, y se sembraron las semillas. Las bandejas se regaron dos veces por día para mantener la humedad y se aplicó un fungicida de amplio espectro (propamocarb clorhidrato $66.90 \%$ en una dosis de $\left.3.5 \mathrm{~cm}^{3} \mathrm{~L}^{-1}\right)$.
Para el trasplante a las platabandas, se hicieron hoyos de $0.15 \mathrm{~m}$ de profundidad a una distancia de $0.60 \mathrm{~m}$ entre plantas, luego se trasplanto una planta por hoyo.

A los 8 y 30 días después del trasplante se aplicó un fungicida sistémico (Metalaxyl-M+Mancozeb $70 \mathrm{~g}$ $15 \mathrm{~L}^{-1}$ ), para el control de del mildiu velloso (Pseudoperonospora cubensis).

A 10 días después del trasplante se aplicó el Acetamiprid (40 g $15 \mathrm{~L}^{-1}$ de agua) para el control de insectos-plaga, como la mosca blanca (Bemisia tabaci), negrita (Prodiplosis longifila) y pulgones (Myzus persicae). Asimismo, a los 20 y 50 días después del trasplante se aplicó dipel (Bacillus thuringiensis subespecie kurstaki) en una dosis de $80 \mathrm{~g}$ $15 \mathrm{~L}^{-1} \underline{36}$.

Se realizó dos riegos día (mañana y tarde) con un sistema de riego por goteo, según la necesidad del cultivo y las condiciones climáticas de la zona.

La cosecha se realizó a partir de los 80 días del trasplante del cultivo. Se recolectó cinco frutos por cada tratamiento. En esta etapa de poscosecha se hizo las evaluaciones participativas de preferencia en la participaron 10 personas, de los cuales cinco fueron hombres y cinco mujeres (dos personas del mercado de abasto, dos comerciantes, dos del supermercado, dos productores y dos consumidores finales).

Para determinar las variables a ser evaluadas, se validó y seleccionó los principales criterios, utilizando la técnica de orden de preferencia y luego se aplicó la técnica de matriz de preferencia ${ }^{37}$.

Para el análisis económico se consideró los costos que varían ${ }^{\frac{38}{}}$, como el costo de semilla, trasplante, poda, plaguicidas, fertilizantes, aplicaciones plaguicidas, mano de obra y otros.

\section{Resultados \\ Resultados}

55 
Análisis de normalidad y homogeneidad de varianzas. La prueba de kosmogorov-Smirnov $\frac{35}{}$, indicó que no hubo significancia al $\operatorname{Pr}<0.01$ de probabilidad y los coeficientes de variación estuvieron entre 16 a $35 \%$, esto reveló que las variables de respuesta tuvieron distribución normal. Asimismo, el análisis de homogeneidad de varianzas con chi-cuadrada al $\operatorname{Pr}<0.05$ de probabilidad, no expuso significancia, indicando que las medias de varianza fueron homogéneas (Chi-cuadrada<0.1440).

Tabla 3 Análisis de varianza para altura de la planta (AP) y diámetro de tallo (DT)

\begin{tabular}{cccc}
\hline \multirow{2}{*}{ FV } & \multirow{2}{*}{ gl } & \multicolumn{2}{c}{ Cuadrados Medios } \\
\cline { 3 - 4 } & & AP & DT \\
\hline Total & 26 & & \\
Tratamiento & 2 & $1.57 \mathrm{~ns}$ & $9.67^{*}$ \\
Error & 24 & 0.53 & 1.91 \\
CV(\%) & & 29.093 & 31.28 \\
\hline *: Diferencias estadísticas significativas al $\mathrm{P}<0.05$ de probabilidad,
\end{tabular}
ns: no significativo, CV: \% Coeficiente de variación.

Análisis de varianza (ANVA). Para altura de la planta (AP) y diámetro de tallo (DT), expuso que los CV
(\%) están entre 29.09 a $31.28 \%$ (tabla 3). Se observó diferencias significativas en $\mathrm{DT}$ al $\mathrm{P}<0.05$ de probabilidad, esto indicó que al menos uno de los tratamientos fue diferente.

Tabla 4 Análisis de varianza para peso de fruto (PFr) $y$ volumen de fruto (VFr)

\begin{tabular}{cccc}
\hline \multirow{2}{*}{ FV } & \multirow{2}{*}{ gl } & \multicolumn{2}{c}{ Cuadrados Medios } \\
\cline { 3 - 4 } & & PFr & VFr \\
\hline Total & 24 & & \\
Tratamiento & 2 & $0.04 \mathrm{~ns}$ & $864.16 \mathrm{~ns}$ \\
Error & 22 & 0.06 & $856.18 \mathrm{~ns}$ \\
CV(\%) & & 16.21 & 23.82 \\
\hline ns = no significativo & & &
\end{tabular}

El ANVA para Peso (PFr) y Volumen (VFr) de fruto no expusieron diferencias significativas al $\mathrm{P}<0.05$ de probabilidad (tabla 4).

Análisis de medias. El análisis de medias de Duncan al $\mathrm{P}<0.05$ de probabilidad para las variables AP, DT, PFr y VFr (tabla 5) manifestó que para AP hubo diferencias significativas, Champagne fue mejor (2.9 m) que Quetzalí (2.1 m) (tabla 5).

Tabla 5 Prueba del rango múltiple de Duncan

\begin{tabular}{ccccc}
\hline Cultivar & AP $(\mathbf{m})$ & DT $(\mathbf{m m})$ & $\mathbf{P F r}(\mathbf{k g})$ & $\mathbf{V F r}\left(\mathbf{c m}^{\mathbf{3}}\right)$ \\
\hline Champagne & $2.90 \mathrm{a}$ & $5.55 \mathrm{a}$ & $2.08 \mathrm{a}$ & 18698 \\
Vanessa & $2.57 \mathrm{ab}$ & $4.00 \mathrm{~b}$ & $1.78 \mathrm{a}$ & 13697 \\
Quetzalí & $2.12 \mathrm{~b}$ & $4.00 \mathrm{~b}$ & $1.63 \mathrm{a}$ & 14225 \\
\hline
\end{tabular}

Para DT, se observó diferencias significativas entre los híbridos, Champagne fue mejor con $5.5 \mathrm{~mm}$, Quetzalí con $4.0 \mathrm{~mm}$ y Vanessa con $3.5 \mathrm{~mm}$ (tabla 7). Champagne (de pulpa amarilla) fue la que mejor peso y adaptación, reveló en invernadero en la zona de estudio, donde, además, se observó una alta correlación significativa entre VFr y PFr (tabla 6).

Respecto al PFr no hubo diferencias significativas, el cultivar Champagne obtuvo mayor peso $(2.1 \mathrm{~kg})$ respecto a Quetzalí (1.6 kg) (tabla 5).
En relación al VFr, no se observaron diferencias significativas. Champagne obtuvo mayor volumen con $18698 \mathrm{~cm}^{3}$, seguido por Quetzalí $\left(14225 \mathrm{~cm}^{3}\right) \mathrm{y}$ Vanessa $\left(13697 \mathrm{~cm}^{3}\right)$ (tabla 5).

Tabla 6 Análisis de correlación

\begin{tabular}{ccccc}
\hline & AP & DT & PFr & VFr \\
\hline AP & 1.0 & 0.08 & 0.23 & 0.23 \\
DT & & 1.0 & 0.20 & 0.12 \\
PFr & & & 1.0 & $0.81^{* *}$ \\
VFr & & & & 1.0 \\
$* *$ Diferencias altamente significativas al & P $<0.01$ de probabilidad,
\end{tabular}


*= Diferencias estadísticas significativas al $\mathrm{P}<0.05$ de probabilidad ns $=$ no significativo. Altura de la planta (AP),

Diámetro de tallo (DT), Peso de fruto (PFr) y volumen de fruto (VFr)

Análisis de correlación. El análisis de correlación con la prueba de Pearson (tabla 6), no manifestó diferencias significativas al $\mathrm{P}<0.05$ de probabilidad para las variables AP, DT, PFr y VFr. Las variables PFr y VFr, manifestaron correlación alta $(r=0.81)$ y alta significativa al $\mathrm{P}<0.01$ de probabilidad, denotando que a mayor VFr hubo mayor PFr.

Análisis participativo en poscosecha. El análisis de Kruskal \& Wallis $\underline{35}$ (tabla 7), mostró diferencias altamente significativas para el color de la piel (CPiel) y significativa para venta al mercado (VMerc).
El cultivar Champagne, fue mejor para CPiel. Para CPulpa, SFr y DFr, no se observaron diferencias significativas. Vanessa tuvo mejor aceptación de preferencia por los participantes. Para el OFr, hubo diferencias significativas, siendo los híbridos Vanessa y Quetzalí de mejor preferencia.

El análisis de Kruskal \& Wallis ${ }^{35}$ para las variables CPiel, TFr, CPulpa, SFr, DFr, OFr y VMerc (tabla 8) manifestó diferencias significativas al $\mathrm{P}<0.05$ de probabilidad para CPiel y $\mathrm{TFr}$, denotándose una mayor preferencia para las mujeres.

Hubo diferencias significativas al $\mathrm{P}<0.05$ de probabilidad para VMerc entre ambos sexos y las mujeres identificaron con mejores atributos para venta al mercado a Quetzalì, seguido de Vanessa y Champagne (tabla 8 ).

Tabla 7 Test de Kruskal-Wallis para determinar la percepción sobre los híbridos Quetzalí, Champagne y Vanessa

\begin{tabular}{lccccccc}
\hline Tratamiento & CPiel & TFr & CPulpa & SFr & DFr & OFr & VMerc \\
\hline Quetzalí & 18.87 & 19.4 & 16.0 & 18.7 & 18.7 & 19.1 & 22.2 \\
Champagne & 19.08 & 22.3 & 17.9 & 14.5 & 14.5 & 12.9 & 15.4 \\
Vanessa & 17.54 & 13.8 & 21.6 & 22.3 & 22.3 & 21.5 & 17.8 \\
\hline Chi-cuadrada & 0.17 & 4.49 & 2.06 & 3.81 & 3.81 & 7.01 & 2.90 \\
gl & 2 & 2 & 2 & 2 & 2 & 2 & 2 \\
Pr $>$ Chi-cuadrado & 0.92 & 0.10 & 0.36 & 0.15 & 0.15 & $0.03 *$ & 0.23 \\
\hline \multicolumn{2}{l}{ **: Altamente significativo al P<0.01 de probabilidad, *: Significativo al P<0.05 de probabilidad, CPiel, Color de piel, TFr: } \\
\multicolumn{2}{l}{ Tamaño de fruto, CPulpa: Color de pulpa, SFr: Sabor de fruto, Ofr: Olor de fruto, VMerc: Venta al mercado. }
\end{tabular}

Tabla 8 Test de Kruskal-Wallis para percepción por sexo (hombre, mujer) sobre los híbridos Quetzalí, Champagne y Vanessa en poscosecha

\begin{tabular}{cccccccc}
\hline Sexo & CPiel & TFr & CPulpa & SFr & DFr & OFr & VMerc \\
\hline Mujer & 22.98 & 22.33 & 21.27 & 20.97 & 20.97 & 20.11 & 22.69 \\
Hombre & 14.11 & 14.67 & 15.72 & 16.03 & 16.02 & 16.88 & 14.30 \\
\hline Chi-cuadrada & 6.90 & 5.29 & 2.96 & 2.32 & 2.32 & 0.97 & 6.44 \\
Gl & 1 & 1 & 1 & 1 & 1 & 1 & 1 \\
Pr $>$ Chi-cuadrado & $0.0086^{* *}$ & $0.02 *$ & 0.09 & 0.13 & 0.13 & 0.32 & $0.01 *$ \\
\hline **: Altamente significativo al P<0.01 de probabilidad, *: Significativo al P<0.05 de probabilidad, CPiel: Color de piel,
\end{tabular}

TFr: Tamaño de fruto, CPulpa: Color de pulpa, SFr: Sabor de fruto, Ofr: Olor de fruto, VMerc: Venta al mercado.

Tabla 9 Rentabilidad de los tratamientos. Jipijapa 2019

\begin{tabular}{|c|c|c|c|c|c|c|c|c|c|}
\hline Cultivar & $\mathrm{NP}\left(1000 \mathrm{~m}^{2}\right)$ & P/fruto (Kg) & PT (Kg) & Precio/Kg (USD) & BB $\left(1000 \mathrm{~m}^{2}\right)$ & Costo $\left(1000 \mathrm{~m}^{2}\right)$ & BN (USD) Supermercado & Relación B/C & Rentabilidad \\
\hline Champagne & 880 & 2.08 & 1830.40 & 0.5 & 915.20 & 371.15 & 544.05 & 1.47 & Rentable \\
\hline Vanessa & 880 & 1.76 & 1548.80 & 0.5 & 774.40 & 371.15 & 403.25 & 1.09 & Rentable \\
\hline Quetzalí & 880 & 1.63 & 1434.40 & 0.5 & 717.20 & 371.15 & 346.05 & 0.93 & No Rentable \\
\hline
\end{tabular}


Análisis del Beneficio/Costo de los tratamientos. El análisis de rentabilidad expresó que los tratamientos tuvieron una relación $\mathrm{B} / \mathrm{C}>1$. El híbrido Champagne expuso mayor rentabilidad con USD 544.05, respecto de Vanessa (USD 403.25) y Quetzalí (USD 346.05) (tabla 9).

\section{Discusión}

Uno de los híbridos que mejor comportamiento tuvo fue el híbrido Champagne, que es diploide $\frac{38}{}$, tiene plantas vigorosas, frutos redondos, color verde claro con estrías verdes oscuras, pulpa color amarillo, crujiente, muy dulce y de excelente sabor, con peso promedio de 5 a $6 \mathrm{~kg}$, muy buena para la poscose$\mathrm{cha}^{\frac{39}{}}$. Cruz-Quintanilla ${ }^{40}$, indica que, en el valle de Moquegua, Perú, este híbrido fue precoz, obteniendo frutos de buena calidad a los 64 días después del trasplante. En nuestra investigación iniciamos la cosecha a los 80 días después del trasplante.

En cambio, Quetzalí un hibrido diploide ${ }^{\frac{38}{}}$, su fruto pesa de 3 a $5 \mathrm{~kg}$, es redonda y muy uniforme, se cosecha a los 80 a 85 días. Color de la cáscara verde intenso con estrías clara, aspecto elegante, pulpa atractiva, firme, de color rojo, con pepitas muy pequeñas y un excelente sabor, alto contenido de azucares. Con resistencia moderada a la raza 1 de antracnosis $\underline{39}$.

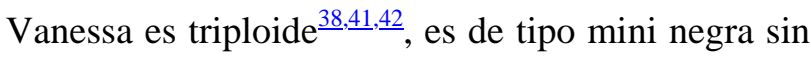
semillas, apta para clima cálido, son plantas vigorosas compactas de alta adaptabilidad y equilibradas con relación a fructificación, inicia su producción a los 65 días. Frutos redondos ligeramente ovalados, sin semillas de 2.5 a $3 \mathrm{~kg}$ en promedio, pulpa de color rojo intenso con excelente contenido de azuca- res, con grosor de la corteza de 9 a $13 \mathrm{~mm}$ y dureza de pulpa de 0.9 a $1 \mathrm{~kg}$.

Se observó que la producción de plántulas para invernadero fue de vital importancia para lograr mejores rendimientos, aspecto corroborado por Danial ${ }^{43}$, quien menciona que el desarrollo, el crecimiento y la producción de los frutos se ven afectada por la calidad de la planta que será trasplantada a campo.

Diversas investigaciones realizadas en la provincia Manabí20-22 ${ }^{2}$, señalaron la importancia de evaluar nuevos genotipos bajo las condiciones de cultivo en estas zonas. Se logró evaluar cerca de 30 híbridos de los cuales varios ya están siendo utilizadas por los agricultores, pero todas estas evaluaciones fueron realizadas bajo condiciones de campo. En nuestro estudio, hemos evaluado los híbridos bajo condiciones de invernadero, esto le da una ventaja competitiva, por la calidad de fruto que se obtiene y el número de cosechas que se podría obtener por año.

La participación de hombres y mujeres en la evalua-

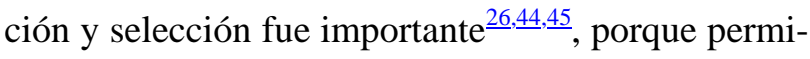
tió evaluar y seleccionar los híbridos con mejor aprobación para el mercado. Esto es fundamental a la hora de difundir un cultivar como lo sugieren diversos investigadores $\underline{26,44,45}$.

La caracterización morfológica de plantas y frutos a través de los caracterizadores morfologicos de INASE $^{32}$, mostró que coinciden con las caracterizaciones de Alaska ${ }^{29}$.

\section{Fuente de financiamiento}

Grant PROG-003-PROY-001-DIP-2017 a JGO, de la Universidad Estatal del Sur de Manabí. 


\section{Conflictos de intereses}

Los autores declaran que esta investigación fue realizada en la Universidad Estatal del Sur de Manabí (Cantón Jipijapa) y no presenta conflictos de interés.

\section{Agradecimientos}

Los autores agradecen a la Universidad Estatal del Sur de Manabí (UNESUM) por el financiamiento y apoyo logístico para realizar las actividades del proyecto financiado por la Universidad Estatal del Sur de Manabí (UNESUM), Ecuador (Grant PROG003-PROXY-001-DIP-2017). Asimismo, agradecemos a todos nuestros estudiantes de la Carrera Agropecuaria, que a lo largo del proyecto se fueron involucrando de manera comprometida y responsable en las diferentes actividades realizadas.

\section{Consideraciones éticas}

La aprobación de la investigación por la Dirección de Investigación y Posgrado, el Comité de Ética, y el Comité de Investigación de la Carrera de Agropecuaria de la Universidad Estatal del Sur de Manabí (UNESUM), (Cantón Jipijapa), se siguió las pautas establecidas por estas instancias.

\section{Aporte de los autores en el artículo}

Julio Gabriel-Ortega, Planeación del experimento, análisis estadístico y sistematización e interpretación de la información. Nora Barahona-Cajape, Toma de datos y sistematización e interpretación de la información. Gema Burgos-López, Toma de datos y sistematización e interpretación de la información. Fernando Ayón-Villao, Planeación del expe- rimento, revisión del artículo. Washington Narváez-

Campana, Planeación del experimento, revisión del artículo. Máximo Vera Tumbaco, Planeación del experimento, revisión del artículo.

\section{Literatura citada}

1. Whitaker TW, Davis GN. Cucurbits: Botany, cultivation, and utilization. J Assoc Off Anal Chem 1962; 45(4):1052. DOI: https://doi.org/10.1093/ jaoac/45.41052

2. Robinson RW, Decker Walters DS. Cucurbits crop production science in horticulture series, Wallingford, U.K.: CAB International; 1997. p. 65-7, 152-62.

3. Paris HS. Origin and emergence of the sweet dessert watermelon, Citrullus lanatus. Ann Bot 2015;116(2):133-48. DOI: https://doi.org/10.1093/aob/mcv077

4. Maynard D, Maynard DN. Cucumbers, melons, and watermelons. In: Kiple KF, Ornelas KC, editors. The Cambridge world history of food. Cambridge: Cambridge University Press; 2000. p. 298-313.

DOI:

https://doi.org/10.1017/CHOL978052

1402149.032

5. Maggs Kölling GL, Madsen S, Christiansen JL. A phenetic analysis of morphological variation in Citrullus lanatus in Namibia. Genet Resour Crop Evol 2000;47:385-93. DOI: https://doi.org/10.10 23/A:1008751319879

6. Levi A, Thomas CE, Keinath AP, Wehner TC. Estimation of genetic diversity among Citrullus accessions using RAPD markers. Acta Hortic 2000;510:385-90. DOI: https://doi.org/10.17660/ ActaHortic.2000.510.61 
7. Levi A, Thomas CE, Keinath AP, Wehner TC. Genetic diversity among watermelon (Citrullus lanatus and Citrullus colocynthis) accessions. Genet Resour Crop Evol 2001;48:559-66. DOI: https:// doi.org/10.1023/A:1013888418442

8. Dane F, Lang P, Bakhtiyarova R. Comparative analysis of chloroplast DNA variability in wild and cultivated Citrullus species. Theo Appl Genet 2004;108(5):958-66.

DOI: https://doi.org/10.1007/s00122-003-1512-9

9. Hwang J, Kang J, Byeonggu S, Kim K, Younghoon P. Genetic diversity in watermelon cultivars and related species based on AFLPs and EST-SSRs. Not Bot Horti Agrobot Cluj Napoca 2011;39(2):285-92.

DOI:

https://doi.org/10.15835/nbha3926382

10.Nimmakayala P, Abburi VL, Bhandary A, Abburi L, Vajja VG, Reddy R, et al. Use of Vera Code 384-plex assays for watermelon diversity analysis and integrated genetic map of watermelon with single nucleotide polymorphisms and simple sequence repeats. Mol Breed 2014;34:537-48. DOI: https://doi.org/10.1007/s11032-014-0056-9

11.Nimmakayala P, Levi A, Abburi L, Abburi VL, Tomason YR, Saminathan T, et al. Single nucleotide polymorphisms generated by genotyping by sequencing to characterize genome-wide diversity, linkage disequilibrium, and selective sweeps in cultivated watermelon. BMC Genomics 2014; 15(1):767. DOI: https://doi.org/10-1186/147121 64-15-767

12.Wehner TC, Shetty NV, Elmstrom GW. Breeding and seed production. In: Maynard DN, editors. Characteristics, production, and marketing. Alexandria: American Society for Horticultural Sci; 2001. p. 27-73.
13.Wehner TC. Watermelon. In: Prohens J, Nuez F, editors. Vegetables I. Handbook of plant breeding. New York: Springer; 2008. p. 381-418. DOI: https://doi.org/10.1007/978-0-387-30443-4_12

14.Food and Agriculture Organization of the United Nations [Internet]. Statistics; 2019 [citado 26 de octubre de 2019]. Recuperado a partir de: https:// faostat.fao.org/site/339/default.aspx

15.Albán Wong G, Arnao Franco C, Mejía Coronel MT. Proyecto de producción de sandía para exportación en la península de Santa Elena [tesis maestría]. [Carolina del Sur]: Escuela Superior Politécnica del Litoral; 2009 [citado 26 de octubre de 2019]. Recuperado a partir de: https://www.ds pace.espol.edu.ec/bitstream/123456789/644/1/11 69.pdf

16.Fundación de Ayuda Social Ecuador Solidario. Informe Final del Proyecto "Producción ecológica y comercialización de sandía y cebolla en Canta Gallo del Cantón Jipijapa". Cofinanciado por el PMRC [Internet]. Jipijapa: Acuerdo Ministerial $n^{\circ}$ 0034; 2006 [citado 15 de octubre de 2019]. 254 p. BID No.: 1531/OC-EC. Recuperado a partir de: https://docplayer.es/25749371Informe-final-del-proyecto-produccion-ecologi ca-y-comercializacion-de-sandia-y-cebolla-encanta-gallo-del-canton-jipijapa-cofinanciado-porel-pmrchtml

17.Gabriel Ortega J, Pereira Murillo E, Ayón Villao F, Castro Piguave C, Delvalle García I, Castillo JA. Development of an ecological strategy for the control of downy mildew (Pseudoperonospora cubensis) in cucumber cultivation (Cucumis sativus L.). Bionatura 2020b;5(2):1101-05. https:// doi.org/10.21931/RB/2020.05.02.3 
18.Janick J, editor. Horticultura Científica e Industrial. Madrid: 6ta tirada, Editorial Mundi prensa; 2002. 337 p.

19.Cantos Loor JF, Giler Meza RI, Mendoza Cedeño L (dir). Comportamiento agronómico de ocho híbridos de sandía (Citrullus lanatus schard.) en el campus de la ESPAM MFL. 2011 [tesis licenciatura]. [Calceta]: Escuela Superior Politécnica Agropecuaria de Manabí Manuel Félix López; 2012 [citado 16 de enero de 2021]. Recuperado a partir de: http://repositorio.espam.edu.ec/xmlui/ handle/42000/22

20.Barcia G, Torres C. Respuesta de cinco híbridos de sandía rayada (Citrullus vulgaris Schard) a tres distanciamientos de siembra en época seca bajo las condiciones del valle del río Portoviejo [tesis licenciatura]. [Manabí]: Universidad Técnica de Manabí; 2007 [citado 16 de enero de 2021]

21.Salazar C. Comportamiento Agronómico de 10 genotipos de sandía sin semillas en el Valle del Río Portoviejo [tesis licenciatura]. [Manabí]: Universidad Técnica de Manabí; 2002 [citado 16 de enero de 2021].

22. Mendoza L. Comportamiento Agronómico de 18 híbridos de Sandía en la zona de influencia del río Canuto [tesis licenciatura]. [Manabí]: Universidad Técnica de Manabí; 2004 [citado 16 de enero de 2021].

23. Soleri D, Cleveland DA. Breeding for quantitative variables. Part 1: Farmers' and scientists' knowledge and practice in variety choice and plant selection. In: Ceccarelli S, Guimarães EP, Weltzien E, editors. Plant Breeding and Farmers Participation [Internet]. Rome: Food and Agriculture Organization of the United Nations; 2009. p. 323-366. Recuperado a partir de: http://www.fao. org/3/i1070e/i1070e.pdf\#page $=337$
24.Gabriel J, Oros R, Nisttahusz S, Rodríguez F, Mendoza O. Experiencia piloto del cambio varietal en los mercados de papa con aptitud para la industria en Bolivia. Rev Latinoam Papa 2017; 21(1):93-119. DOI: https://doi.org/10.37066/rlap. v21i1.267

25.Vernooy R, Shrestha P, Ceccarelli S, Ríos H, Song Y, Humphries S. Towards new roles, responsibilities and rules: the case of participatory plant breeding. In: Ceccarelli S, Guimarães EP, Weltzien E, editors. Plant Breeding and Farmers Participation [Internet]. Rome: Food and Agriculture Organization of the United Nations; 2009. p. 613-28. Recuperado a partir de: https://www.academia.edu/19277831/Towards n ew_roles_responsibilities_and_rules_the_case_of _participatory_plant_breeding

26. Thiele G, Gadner G, Torrez R, Gabriel J. Farmer involvement in selecting new varieties: Potatoes in Bolivia. Exp Agric 1997;33(03):275-90. DOI: https://doi.org/10.1017/S0014479797003098

27. Gabriel J, Oros R, Nisttahusz S, Rodríguez F, Mendoza O. Experiencia piloto del cambio varietal en los mercados de papa con aptitud para la industria en Bolivia. Rev Latinoam Papa 2017; 21(1):93-119. https://doi.org/10.37066/ralap.v21 $\underline{\mathrm{i} 1.267}$

28. Secretaria Nacional de Planificación y Desarro1lo. Plan de Ordamiento y Desarrollo Territorial de Puerto Cayo 2015 [Internet]. Puerto Cayo: Secretaria Nacional de Planificación y Desarrollo; 2015 [citado 22de octubre de 2019]. 231 p. Recuperado a partir de: http://app.sni.gob.ec/sni$\underline{\text { link/sni/PORTAL_SNI/data_sigad_plus/sigadplu }}$ sdocumentofinal/1360043010001_PDOT\%20PU ERTO\%20CAYO\%20FINAL_31-10-2015_0004-31.pdf 
29.Sandia híbrida Catira [Internet]. Alaska S.A.Importadora. 2020 [citado 5 de marzo de 2020]. Recuperado a partir de: https://www.imporaalas ka.com/sandias

30.Gabriel J, Castro C, Valverde A, Indacochea B, Diseños experimentales: Teoría y práctica para experimentos agropecuarios. Grupo COMPAS, Universidad Estatal del Sur de Manabí (UNESUM), Jipijapa, Ecuador. 2017. 116 p. Recuperado a partir de http://142.93.18.15:8080 /jspui/handle/123456789/116

31. Moreno JM. Fórmulas y volúmenes para la determinación de áreas y volúmenes. Guiniguada 2000;9(8/9):291-3187.

32.Descriptores de cultivares. Hortícolas [Internet]. Instituto Nacional de Semillas-Descripción de cultivar de Sandía. 2018 [citado 5 de marzo de 2019]. Recuperado a partir de: https://www.argentina.gob.ar/variedadesvegetales/descriptor-de-cultivares/horticolas

33.Barahona Cajape NL, Gabriel Ortega J (dir). Evaluación de híbridos de sandía (Citrullus lanatus L.) bajo invernadero en Puerto la Boca, Jipijapa [tesis licenciatura]. [Jipijapa]: Universidad Estatal del Sur de Manabí; 2019. [citado 16 de octubre de 2019]. Recuperado a partir de http://repositorio.unesum.edu.ec/handle/53000/19 $\underline{94}$

34.SAS University [Internet]. An Introduction to SAS University Edition; 2019 [citado 26 de octubre de 2019]. Recuperado a partir de: https:// www.oreilly.com/library/view/an-introductionto/9781629600079/

35.Berlanga Silvente V, Rubio Hurtado MJ. Clasificación de pruebas no paramétricas. Cómo aplicarlas en SPSS. REIRE 2012;5(2):101-13. DOI: https://doi.org/0.1344/reire2012.5.2528
36. Bio Huerto [Internet]. Insecticida larvicida biológico BT Dipel DF. 2019 [citado 5 de marzo de 2019]. Recuperado a partir de: https://www. biohuerto.es/insecticida-profesional/6323insecticida-larvicida-biologico-bt-dipel-df$\underline{\mathrm{kg} . \mathrm{html}}$

37.Gandarillas E, Almanza J. Cómo escoger técnicas para evaluar alternativas tecnológicas con la participación de agricultores [Internet]. Cochabamba: Promoción e Investigación de Productos Andinos, Fundación Proinpa; 2002 [citado 26 de octubre de 2019]. Ficha Técnica $N^{\circ} 7$. Recuperado a partir de: https://www.proinpa.org/tic/pdf/Metodologias\%2 Oparticipativas/Evaluacion/Como\%

20escoger\%20tecnicas\%20para\%20eva luar\%20 tecnologias\%20con\%20agricultores.pdf

38.Centro Internacional de Mejoramiento de Maíz y Trigo. CIMMYT Publications Repository [Internet]. La formulación de recomendaciones a partir de datos agronómicos: un manual metodológico de evaluación económica; 1988. [citado 19 de octubre de 2019]. Recuperado a partir de: https://repository.cimmyt.org/xmlui/bitstream/ha ndle/108 83/1063/9031.pdf

39.Jamatia J, Choudhary H, Basavaraj B. Morphological studies on interspecific hybrids between Citrullus lanatus L. and its wild species. Int J Chem Stud 2018;6(4):199-202.

40.Cruz Quintanilla HO. Rendimiento de quince cultivares de sandía (Citrullus lanatus Thunb), en el valle de Moquegua [tesis licenciatura]. [Tacna]: Universidad Nacional Jorge Basadre Grohmann; 2010 [citado 26 de octubre de 2019]. Recuperado a partir de: http://repositorio.unjbg. $\underline{\text { edu.pe/bitstre }}$ 
am/handle/UNJBG/541/TG0413.pdf? sequence=1 \&isAllowed $=\mathrm{y}$

41.Ahmad I, Hussain T, Nafees M, Maryam Jamil M, Ashraf I, Fachar-u-Zaman Akhtar M, et al. Morphological dissimilarity between tetrapoloid and diploid watermelon (Citrullus lanatus Thunb.). World Appl Sci J 2013;21(6):858-61. DOI: https: //doi.org/10.5829/idosi.wasj.2013.21. $\underline{6.169}$

42. Hawkins LK, Dane F, Kubisiak TL, Rhodes BB, Jarret RL. Linkage mapping in a watermelon population segregating for Fusarium wilt resistance. J Amer Soc Hort Sci 2001;12683):34450. DOI: https://doi.org/10.21273/JASHS.126.3. $\underline{344}$

43.Danial D. Gestión de los recursos y manejo de cultivos. En: Gabriel J, Crespo M, Danial D, editores. Curso sobre producción de hortalizas de alta calidad para el mercado interno. Cochabamba: Fundación para la Promoción de Producto Andinos-PROINPA; 2013. p. 41-2. DOI: https://doi.org/10.13140/2.1.1064.3526

44.Ríos Labrada H. Participatory seed diffusion: experiences from the field. In: Ceccarelli $\mathrm{S}$, Guimarães EP, Weltzien E, editors. Plant Breeding and Farmers Participation [Internet]. Rome: Food and Agriculture Organization of the United Nations; 2009. p. 589-612. Recuperado a partir de: http://www.fao.org/3/i1070e/i1070e06.pdf
45.Annicchiarico P. Coping with and exploiting genotype-by-environment interactions. In: Ceccarelli S, Guimarães EP, Weltzien E, editors. Plant Breeding and Farmers Participation [Internet]. Rome: Food and Agriculture Organization of the United Nations; 2009. p. 519-64. Recuperado a partir de: http://www.fao.org/3/i1070e/i1070e05. pdf

Nota del Editor:

Journal of the Selva Andina Research Society (JSARS) se mantiene neutral con respecto a los reclamos jurisdiccionales publicados en mapas y afiliaciones institucionales. 